\title{
High-Resolution Cone-Beam Computed Tomography is a Fast and Promising Technique to Quantify Bone Microstructure and Mechanics of the Distal Radius
}

Citation for published version (APA):

Mys, K., Varga, P., Stockmans, F., Gueorguiev, B., Neumann, V., Vanovermeire, O., Wyers, C. E., van den Bergh, J. P. W., \& van Lenthe, G. H. (2021). High-Resolution Cone-Beam Computed Tomography is a Fast and Promising Technique to Quantify Bone Microstructure and Mechanics of the Distal Radius. Calcified Tissue International, 108(3), 314-323. https://doi.org/10.1007/s00223-020-00773-5

Document status and date:

Published: 01/03/2021

DOI:

10.1007/s00223-020-00773-5

Document Version:

Publisher's PDF, also known as Version of record

Document license:

Taverne

Please check the document version of this publication:

- A submitted manuscript is the version of the article upon submission and before peer-review. There can be important differences between the submitted version and the official published version of record.

People interested in the research are advised to contact the author for the final version of the publication, or visit the DOI to the publisher's website.

- The final author version and the galley proof are versions of the publication after peer review.

- The final published version features the final layout of the paper including the volume, issue and page numbers.

Link to publication

\footnotetext{
General rights rights.

- You may freely distribute the URL identifying the publication in the public portal. please follow below link for the End User Agreement:

www.umlib.nl/taverne-license

Take down policy

If you believe that this document breaches copyright please contact us at:

repository@maastrichtuniversity.nl

providing details and we will investigate your claim.
}

Copyright and moral rights for the publications made accessible in the public portal are retained by the authors and/or other copyright owners and it is a condition of accessing publications that users recognise and abide by the legal requirements associated with these

- Users may download and print one copy of any publication from the public portal for the purpose of private study or research.

- You may not further distribute the material or use it for any profit-making activity or commercial gain

If the publication is distributed under the terms of Article $25 \mathrm{fa}$ of the Dutch Copyright Act, indicated by the "Taverne" license above, 


\title{
High-Resolution Cone-Beam Computed Tomography is a Fast and Promising Technique to Quantify Bone Microstructure and Mechanics of the Distal Radius
}

\author{
Karen Mys ${ }^{1,2}$ - Peter Varga ${ }^{2} \cdot$ Filip Stockmans $^{3} \cdot$ Boyko Gueorguiev $^{2} \cdot$ Verena Neumann $^{2} \cdot$ Olivier Vanovermeire $^{4}$. \\ Caroline E. Wyers ${ }^{5,6}$. Joop P. W. van den Bergh ${ }^{5,6,7}$. G. Harry van Lenthe ${ }^{1}$
}

Received: 5 May 2020 / Accepted: 27 October 2020 / Published online: 16 January 2021

(c) Springer Science+Business Media, LLC, part of Springer Nature 2021

\begin{abstract}
Obtaining high-resolution scans of bones and joints for clinical applications is challenging. HR-pQCT is considered the best technology to acquire high-resolution images of the peripheral skeleton in vivo, but a breakthrough for widespread clinical applications is still lacking. Recently, we showed on trapezia that CBCT is a promising alternative providing a larger FOV at a shorter scanning time. The goals of this study were to evaluate the accuracy of CBCT in quantifying trabecular bone microstructural and predicted mechanical parameters of the distal radius, the most often investigated skeletal site with HR-pQCT, and to compare it with HR-pQCT. Nineteen radii were scanned with four scanners: (1) HR-pQCT (XtremeCT, Scanco Medical AG, @ (voxel size) $82 \mu \mathrm{m}$ ), (2) HR-pQCT (XtremeCT-II, Scanco, @60.7 $\mu \mathrm{m}$ ), (3) CBCT (NewTom 5G, Cefla, @75 $\mu \mathrm{m}$ ) reconstructed and segmented using in-house developed software and (4) microCT (VivaCT40, Scanco, @ $19 \mu \mathrm{m}$ — gold standard). The following parameters were evaluated: predicted stiffness, strength, bone volume fraction (BV/ TV) and trabecular thickness (Tb.Th), separation (Tb.Sp) and number (Tb.N). The overall accuracy of CBCT with in-house optimized algorithms in quantifying bone microstructural parameters was comparable $\left(R^{2}=0.79\right)$ to XtremeCT $\left(R^{2}=0.76\right)$ and slightly worse than XtremeCT-II $\left(R^{2}=0.86\right)$ which were both processed with the standard manufacturer's technique. CBCT had higher accuracy for BV/TV and Tb.Th but lower for Tb.Sp and Tb.N compared to XtremeCT. Regarding the mechanical parameters, all scanners had high accuracy $\left(R^{2} \geq 0.96\right)$. While HR-pQCT is optimized for research, the fast scanning time and good accuracy renders CBCT a promising technique for high-resolution clinical scanning.
\end{abstract}

Keywords CBCT $\cdot \mathrm{HR}-\mathrm{pQCT} \cdot \mathrm{Bone}$ parameters

Karen Mys

Karen.Mys@aofoundation.org

1 Biomechanics Section, Mechanical Engineering, KU Leuven, Leuven, Belgium

2 AO Research Institute Davos, Davos, Switzerland

3 Muscles \& Movement, Department of Development and Regeneration, KU Leuven Campus Kulak, Kortrijk, Belgium

4 Department of Radiology, AZ Groeninge, Kortrijk, Belgium

5 Department of Internal Medicine, VieCuri Medical Center, Venlo, The Netherlands

6 NUTRIM School for Nutrition and Translational Research in Metabolism, Maastricht University, Maastricht, The Netherlands

7 Rheumatology, Department of Internal Medicine, Maastricht University Medical Centre, Maastricht, The Netherlands

\section{Introduction}

Osteoporosis is a multi-factorial disorder of reduced bone strength and increased fragility, resulting from decrease in bone mass and deterioration of bone micro-architecture [1] . Osteoporosis induces direct medical costs over 37 billion Euro/year in Europe [2]. Quantification of bone mineral density (aBMD) using dual-energy X-ray absorptiometry (DXA) combined with clinical risk factors (e.g. age, weight, gender, smoking history, alcohol use and fracture history) is the gold standard to assess the risk of osteoporosis and subsequent fragility fractures [3], which is clinically available as the Fracture Risk Assessment (FRAX) tool [4]. However, 50\% of all fractures occur in the large proportion of the population diagnosed with osteopenia, which has, following the current evaluation with FRAX, only a modest fracture risk $[5,6]$. Therefore, it is important to take other bone-related 
factors into account, such as trabecular and cortical parameters as well as mechanical parameters, which can be assessed and quantified in vivo with high-resolution imaging systems.

The state-of-the-art technique to quantify bone microstructural parameters is high-resolution peripheral quantitative computed tomography (HR-pQCT) [7]. It is also the state-of-the-art to quantify bone mechanical parameters in vivo by making use of microFE simulations. Two imaging systems are currently available (XtremeCT and XtremeCT-II, Scanco Medical AG, Switzerland), which provide a reconstructed voxel size up to $82 \mu \mathrm{m}$ and $60.7 \mu \mathrm{m}$, respectively, with a FOV (stack) of $12.6 \phi \times 0.9 \mathrm{~cm}^{3}$ and 14.0 $\phi \times 1.0 \mathrm{~cm}^{3}$. The rather long scan time $(168 \mathrm{~s}$ for XtremeCT and $120 \mathrm{~s}$ for XtremeCT-II for one stack) increases the risk of motion artefacts and inhibits scanning of a large field of view (FOV) in vivo, which hampers a breakthrough in clinical practice for general applications.

A relatively new alternative imaging technique with a larger field of view is high-resolution cone-beam computed tomography (CBCT) [8]. The top range of the state-ofthe-art CBCT scanners has a high spatial resolution, large FOV, short scanning time and low radiation dosage (e.g. a voxel size of $75 \mu \mathrm{m}$ and a field of view of $12 \times 8 \mathrm{~cm}^{3}$ in 18-36 s). Until today, these scanners are mainly used for dental applications.

Recently, we have demonstrated on trapezia that the image quality of the CBCT device NewTom 5G (Cefla, Italy) [9] can be enhanced to reach an accuracy comparable to HR-pQCT in quantifying bone trabecular parameters [10]. This enhancement consists mainly of an in-housedeveloped Feldkamp-Davis-Kress (FDK) reconstruction and beam-hardening correction algorithm and replaced the reconstruction program of the manufacturer completely. This was combined with an adaptive thresholding technique as segmentation tool and a direct analysis tool (Scanco Medical AG, Switzerland). The manufacturer Cefla (Italy) does not suggest a segmentation technique nor an analysis tool. Yet, not the trapezium but the distal radius is the skeletal site that is most often investigated with HR-pQCT scanners, given its confirmed relevance in osteoporosis research and for prediction of fragility fractures [11]. Therefore, the aims of this study were (1) to evaluate the accuracy of the previously developed CBCT-based analysis in quantifying bone microstructural and mechanical parameters of the distal radius and (2) to compare the accuracies of CBCT and HR-pQCT.

\section{Materials and Methods}

\section{Sample Collection}

Nineteen radii (11 right, 8 left) of 14 female and 5 male donors aged between 25 to 93 years (mean \pm SD
$67.9 \pm 16.2$ year) were obtained from Science Care (United States). The donors donated their bodies to science. Only radii fitting in the FOV of the VivaCT40 (Scanco Medical AG, Switzerland-diameter of $39 \mathrm{~mm}$ ) were selected for this study. The samples were stored at $-20^{\circ} \mathrm{C}$ and thawed prior to scanning for $3 \mathrm{~h}$.

\section{Image Acquisition and Embedding}

Following thawing, the radii were first soaked in room temperature water for $30 \mathrm{~min}$ to rewet the tissues. Afterwards, the bones were double vacuum-packed and embedded in a PMMA cylinder (46 mm diameter and $65 \mathrm{~mm}$ height) at $75 \mathrm{~mm}$ measured from the distal end that allowed reproducible positioning in the different scanners. The bone long axis was aligned with three line lasers aligned in different planes to assure centralized vertical positioning within the cylindrical embedding holder (Fig. 1a, b). The centralized alignment was essential for fitting the FOV of the microCT scanner (Fig. 1c). The distal radii were then scanned with four different scanners, by making use of custom sample holders (Fig. 1c): (1) using a HR-pQCT (XtremeCT, Scanco Medical AG, Switzerland) at a voxel size of $82 \mu \mathrm{m}$, (2) using a HR-pQCT (XtremeCT-II, Scanco Medical AG, Switzerland) at a voxel size of $60.7 \mu \mathrm{m}$, (3) using a CBCT (NewTom $5 \mathrm{G}$, Cefla, Italy) scanned following the $75 \mu \mathrm{m}$ protocol of the scanner and reconstructed at a voxel size of $60 \mu \mathrm{m}$ by means of in-house developed software [9] and (4) using a smallanimal microCT scanner (VivaCT40, Scanco Medical AG, Switzerland) at a voxel size of $19 \mu \mathrm{m}$ (Fig. 2). The microCT scanner, having the highest resolution, was used as the gold standard in all further analyses [12].

\section{Selecting Sections and Volume of Interest}

Two adjacent sections of $9 \mathrm{~mm}$ length were selected for each distal radius based on the microCT scans. The first section was selected strictly adjacent to the most proximal point of the subchondral endplate, aligned perpendicular to the long bone axis and termed 'subchondral section' in this study. The second section was selected directly distal to the first layer, and it mimics the measurement area recommended for clinical scanning, termed 'standard section' throughout this study [13].

\section{Image Segmentation}

The XtremeCT, XtremeCT-II and microCT images were segmented following the standard manufacturer's protocol which is for all of them a filtering operation followed by a global threshold. In more detail, for the XtremeCT a Laplace-Hamming filter and for the XtremeCT-II and microCT VivaCT40 a Gaussian filter were proposed by the 


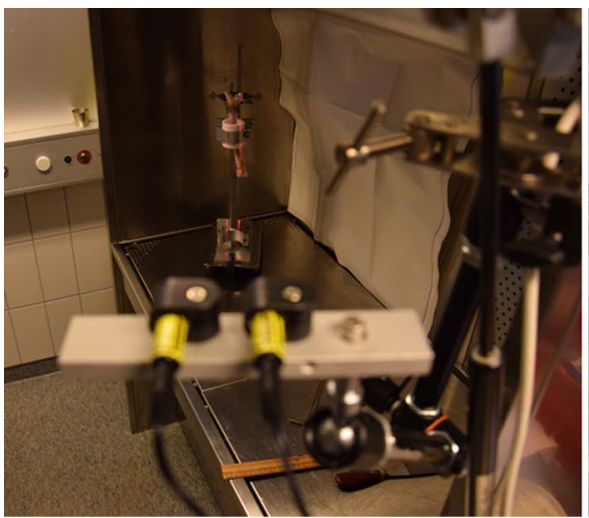

(a)

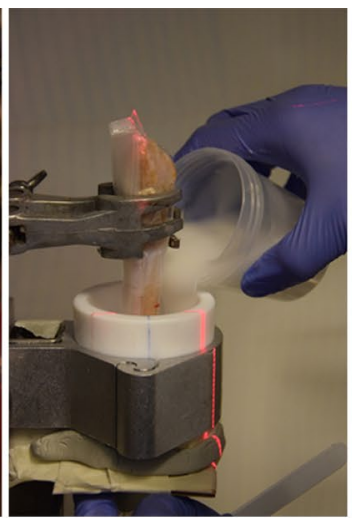

(b)

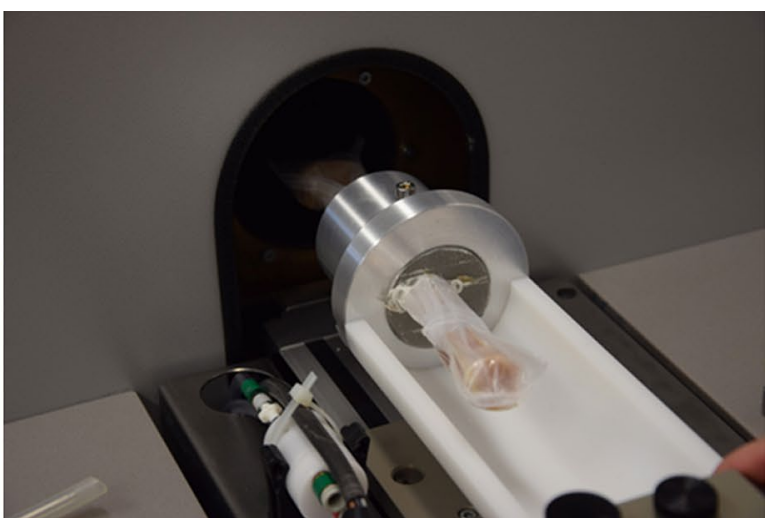

(c)
Fig. 1 Demonstration of the sample preparation steps and scanning. The radii were $\mathbf{a}$ aligned in the centre of a FOV of $3.9 \mathrm{~cm}$ by making use of 3 lasers, $\mathbf{b}$ embedded in a PMMA ring starting $7.5 \mathrm{~cm}$ from the

manufacturer and used in this study with the default settings. The CBCT images were segmented using adaptive thresholding as described in Mys et al. [9]. First, a global pre-segmentation step was performed with a low global threshold and used as input for the adaptive segmentation. To reduce the noise, the pre-segmented volume was masked with a Gaussian filter (sigma of 1) followed by global thresholding with the same low threshold level. In parallel, a high global threshold was applied to select the thick bone parts (e.g. cortical bone) which would be unselected by the adaptive segmentation process. Finally, both segmentations were combined. The low and high global thresholds in the adaptive segmentation technique were optimized in steps of $5 \%$ of the highest grey value to the highest correlation for both subsections together. To avoid overoptimization, the optimization was checked on random subsets of the dataset. The optimization was done separately for three parameter groups. The first group is BV/TV, Tb.Sp and Tb.N, the second group is $\mathrm{Tb}$.Th and the third group are the mechanical parameters. The volume of interest (VOI) corresponding to trabecular bone was selected automatically based on the microCT images using the masking method of Buie et al. [14] as described in more detail in Mys et al. [9].

\section{Calculation of Bone Microstructural Parameters}

Bone volume fraction (BV/TV), trabecular thickness (Tb. $\mathrm{Th})$, trabecular separation (Tb.Sp) and trabecular number (Tb.N) were calculated within the VOI using the Image Processing Language (IPL) software of Scanco. Following the manufacturer's guidelines, the segmented XtremeCT images were analysed using indirect bone microstructural evaluation assuming a parallel plate model, whereas the segmented XtremeCT-II and microCT images were analysed by means of the direct microstructural analysis. For CBCT, for which distal end of the bone and c scanned by making use of scanner-specific holders to allow scanning of all the radii in the same orientation in all scanners. The VivaCT40-holder is shown on the picture

no standard analysis method exists, the same direct microstructural analysis was used.

\section{Calculation of Bone Mechanical Parameters}

Bone stiffness and strength were calculated by means of the microFE analysis software ParOsol on all scans of all scanners. Prior to the analysis, component labelling was applied in Matlab R2017b (The Mathworks, United States) to the segmented images so that only the largest connected part (6-part connectivity) was considered. Each bone voxel of the segmented images was converted to an equally sized brick element in the microFE model. Consequently, the size of the brick elements was scanner-specific and depended on the voxel size of the scan. The bone material was implemented as a homogenous linear elastic material with a Young's modulus of $15 \mathrm{GPa}$ and a Poisson's ratio of 0.3 . As boundary conditions, the most proximal nodes were fixed in all directions and the most distal nodes were displaced with $1 \mathrm{~mm}$ along the longitudinal direction. The boundary conditions, together with the segmented image and the material properties, were directly written in a h5-file. This h5-file could be run directly in the voxel-based microFE-software ParOsol [15]. This technique was used for all scans of all scanners.

The microFE analyses were solved on a Hybrid Cray XC407XC50 on Piz Daint at CSCS (Switzerland) using one or two nodes each consisting of $36 \mathrm{CPU}$ cores. Bone stiffness was calculated by summing the forces at the constraint proximal nodes and dividing it by the applied displacement. Bone strength was calculated using the Pistoia criterion [16]. Specifically, the bone strength was defined as the force at which $6 \%$ of the bone voxels experienced an effective strain equal or larger than $0.7 \%$. 
Fig. 2 The reconstructed images on the left and the corresponding segmented images on the right. a MicroCT VivaCT40 (Scanco Medical AG, Switzerland); b XtremeCT (Scanco Medical AG, Switzerland) image segmented using the standard Scanco technique (Laplace-Hamming filter + fixed threshold); c XtremeCT-II (Scanco Medical AG, Switzerland) image segmented using the standard Scanco technique (Gaussian filter + fixed threshold); $\mathbf{d}$ in-house reconstructed CBCT NewTom 5G (Cefla, Italy) image with beam-hardening correction and segmented using adaptive segmentation
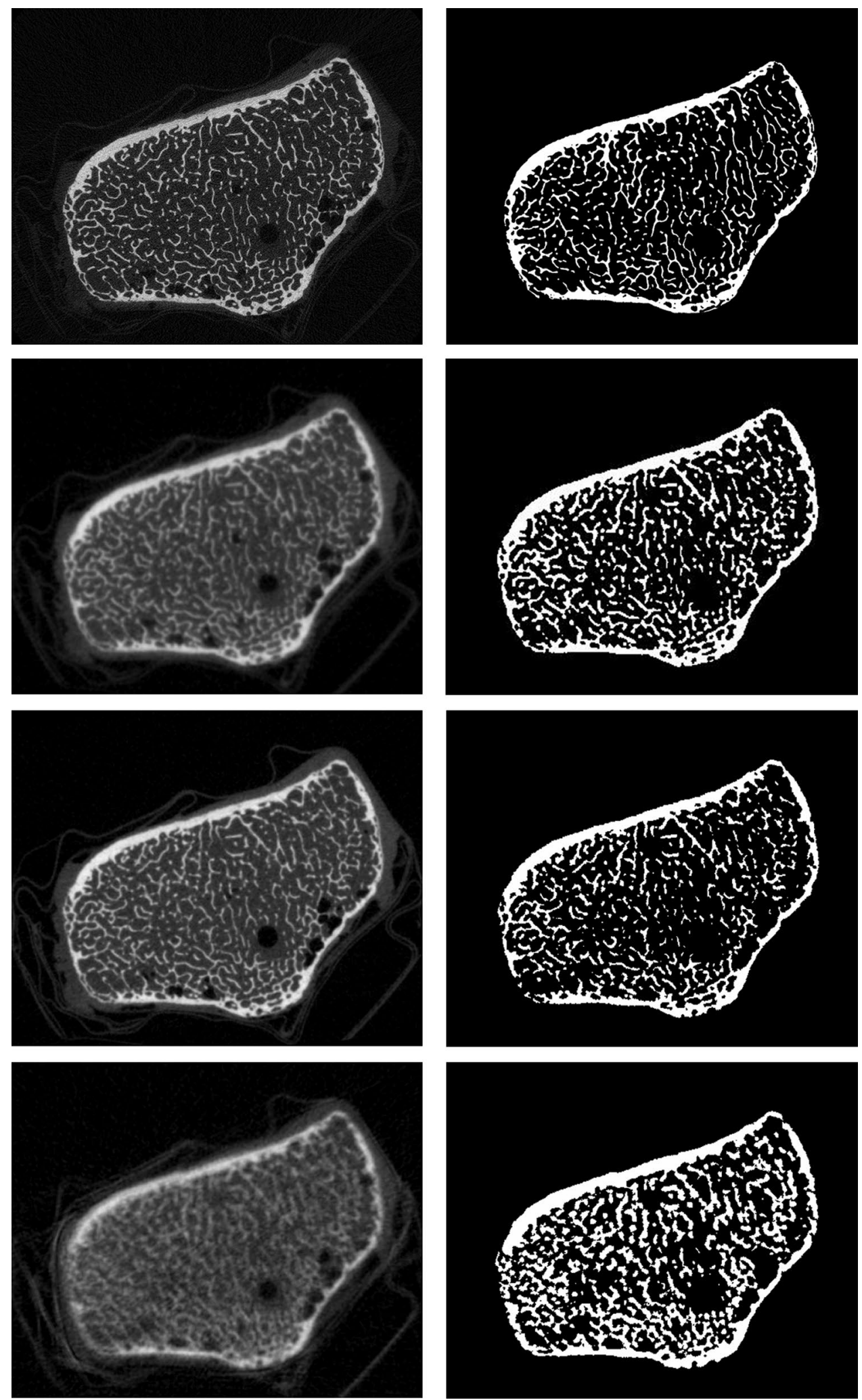

\section{Image Registration}

In order to compare bone parameters of the different scanners, the same VOI needed to be evaluated for the microstructural bone parameters and the same boundary and loading conditions had to be applied on the microFE models. Performing the calculations on registered CT images would have resulted in loss of accuracy, because details 
in the microstructure would have been lost due to resampling and interpolation. To avoid this, the bone VOI mask was transformed for evaluation of the bone microstructural parameters. The corresponding transformation matrices were determined by spatially registering the images of the XtremeCT, XtremeCT-II and CBCT to microCT using the software Amira v6.2 (Thermo Fisher Scientific, USA).

The segmented images were not rotated to generate the microFE models, but the use of the custom sample holders ensured negligible misalignment. For XtremeCT, the maximal axial misalignment with microCT was $2.37^{\circ}$ (mean $1.03^{\circ}$, SD $0.64^{\circ}$ ), for XtremeCT-II $4.07^{\circ}$ (mean $2.19^{\circ}$, SD $0.83^{\circ}$ ) and for $\mathrm{CBCT} 2.78^{\circ}$ (mean $1.98^{\circ}, \mathrm{SD} 0.52^{\circ}$ ).

\section{Statistics}

Accuracy was quantified by comparing the results of the XtremeCT, XtremeCT-II and CBCT scanners with the microCT data via linear regression analysis. Also the intercept, slope and offset as well as the coefficient of determination were calculated against microCT. Offset was calculated as the average difference with the microCT-based value.
Scatter plots and Bland-Altman plots were generated for a visual and quantitative assessment of accuracy. All statistical tests were performed in Matlab R2017b (The Mathworks, United States).

\section{Results}

A summary of the bone microstructural parameters BV/TV, $\mathrm{Tb} . \mathrm{Th}, \mathrm{Tb} . \mathrm{Sp}$ and Tb.N and bone mechanical parameters, stiffness and strength is given in Table 1 . Specifically, the mean and standard deviation of all parameters are listed for microCT. Furthermore, the relative offset, slope, intercept and coefficient of determination of the HR-pQCT and $C B C T$ scanners against microCT are provided. Note that the accuracy of the bone parameters reflects a combination of the scanner and the image processing afterwards on the scan.

\section{Bone Microstructural Parameters}

For all parameters and all scanners significant correlations $(p<0.05)$ were obtained for both bone sections. For the

Table 1 Mean and standard deviation for the microstructural parameters BV/TV, Tb.Th, Tb.Sp and Tb.N and bone mechanical parameters stiffness and strength as determined by microCT for the subchondral section as well as for the standard section

\begin{tabular}{|c|c|c|c|c|c|c|c|c|c|c|c|c|c|c|}
\hline & \multicolumn{2}{|c|}{ MicroCT } & \multicolumn{4}{|c|}{ XtremeCT } & \multicolumn{4}{|c|}{ XtremeCT-II } & \multicolumn{4}{|l|}{ СBCT } \\
\hline & Mean & SD & Intercept & Slope & Offset & $R^{2}$ & Intercept & Slope & Offset & $R^{2}$ & Intercept & Slope & Offset & $R^{2}$ \\
\hline \multicolumn{15}{|l|}{ Subchondral } \\
\hline $\mathrm{BV} / \mathrm{TV}[\%]$ & 16.03 & 3.36 & -0.45 & 0.83 & $-{ }^{\mathrm{a}}$ & 0.88 & -1.84 & 1.25 & 7.85 & 0.96 & -0.62 & 1.86 & 84.44 & 0.92 \\
\hline Tb.Th [mm] & 0.15 & 0.01 & -0.08 & 1.14 & $-{ }^{\mathrm{a}}$ & 0.45 & 0.04 & 1.12 & 25.69 & 0.84 & 0.19 & 0.60 & 53.57 & 0.83 \\
\hline Tb.Sp [mm] & 0.70 & 0.11 & -0.40 & 1.50 & $-^{\mathrm{a}}$ & 0.82 & -0.31 & 1.58 & 9.02 & 0.75 & -0.42 & 1.63 & -5.07 & 0.58 \\
\hline Tb.N [1/mm] & 1.45 & 0.22 & -0.13 & 1.03 & $-^{\mathrm{a}}$ & 0.75 & -0.06 & 0.90 & -14.45 & 0.75 & -0.31 & 1.16 & -0.50 & 0.71 \\
\hline Avrage & & & -0.26 & 1.13 & $-{ }^{\mathrm{a}}$ & 0.73 & -0.54 & 1.21 & 7.03 & 0.83 & -0.29 & 1.31 & 33.11 & 0.76 \\
\hline Stiffness $[\mathrm{kN} / \mathrm{mm}]$ & 77.97 & 27.63 & 6.00 & 1.23 & 35.13 & 0.99 & 1.41 & 1.19 & 1.39 & 0.98 & 4.53 & 1.32 & 57.31 & 0.97 \\
\hline Strength $[\mathrm{kN}]$ & 4.23 & 1.40 & 0.45 & 1.12 & 40.86 & 0.98 & 0.08 & 1.09 & 4.93 & 0.99 & 0.34 & 1.21 & 64.08 & 0.97 \\
\hline Average & & & 3.23 & 1.18 & 38.00 & 0.99 & 0.74 & 1.14 & 3.16 & 0.99 & 2.44 & 1.27 & 60.70 & 0.97 \\
\hline \multicolumn{15}{|l|}{ Standard } \\
\hline $\mathrm{BV} / \mathrm{TV}[\%]$ & 12.45 & 3.89 & -1.65 & 0.83 & $--^{\mathrm{a}}$ & 0.86 & -5.84 & 1.46 & 9.47 & 0.94 & -0.48 & 1.88 & 80.13 & 0.95 \\
\hline Tb.Th [mm] & 0.15 & 0.01 & -0.05 & 0.94 & $--^{\mathrm{a}}$ & 0.58 & 0.04 & 1.17 & 23.91 & 0.85 & 0.18 & 0.67 & 48.89 & 0.69 \\
\hline Tb.Sp $[\mathrm{mm}]$ & 0.82 & 0.18 & -0.16 & 1.17 & $-^{\mathrm{a}}$ & 0.88 & -0.07 & 1.26 & 13.32 & 0.88 & -0.20 & 1.31 & 5.25 & 0.77 \\
\hline Tb.N [1/mm] & 1.26 & 0.27 & 0.04 & 0.93 & $-^{\mathrm{a}}$ & 0.86 & 0.07 & 0.81 & -15.81 & 0.88 & -0.28 & 1.19 & -9.19 & 0.88 \\
\hline Average & & & -0.45 & 0.97 & $-^{\mathrm{a}}$ & 0.80 & -1.45 & 1.17 & 7.72 & 0.89 & -0.20 & 1.26 & 31.27 & 0.82 \\
\hline Stiffness $[\mathrm{kN} / \mathrm{mm}]$ & 101.13 & 33.37 & -1.88 & 1.53 & 17.88 & 0.98 & -24.11 & 1.52 & 9.10 & 0.98 & 0.41 & 1.73 & 24.59 & 0.96 \\
\hline Strength $[\mathrm{kN}]$ & 5.37 & 1.76 & 0.04 & 1.40 & 21.70 & 0.98 & -1.20 & 1.37 & 11.20 & 0.98 & 0.09 & 1.61 & 28.94 & 0.96 \\
\hline Average & & & -0.92 & 1.46 & 19.79 & 0.98 & -12.65 & 1.44 & 10.15 & 0.98 & 0.25 & 1.67 & 26.77 & 0.96 \\
\hline
\end{tabular}

For XtremeCT, XtremeCT-II and CBCT, the slope, intercept, relative offset (in percentage against microCT) and the coefficient of determination $\left(R^{2}\right)$ are given with respect to microCT. For XtremeCT and XtremeCT-II, the standard segmentation techniques were used and for CBCT an adaptive segmentation technique was used

${ }^{a}$ For XtremeCT, the offset is not reported because due to the indirect analysis, the offset is made artificially low and not comparable with the other scanners which are analysed with a direct analysis method 
standard section (Fig. 3), the highest coefficient of determination for BV/TV was obtained for CBCT $\left(R^{2}=0.95\right.$; Table 1). The weakest correlation for CBCT-based data was found for $\mathrm{Tb} . \mathrm{Th}\left(R^{2}=0.69\right)$. The obtained accuracy over all bone microstructural parameters of the standard section of CBCT $\left(R^{2}=0.82\right)$ was slightly better than for XtremeCT $\left(R^{2}=0.80\right)$ and worse than for XtremeCT-II $\left(R^{2}=0.89\right)$. The accuracy of CBCT was higher than XtremeCT for the trabecular thickness $\left(R^{2}=0.69\right.$ for CBCT against $R^{2}=0.58$ for XtremeCT). For Tb.Sp, the opposite was true $\left(R^{2}=0.88\right.$ for XtremeCT against $R^{2}=0.77$ for CBCT). The accuracy of CBCT versus XtremeCT-II was similar for BV/TV and for Tb.N, and slightly lower for Tb.Th and for Tb.Sp (Table 1).

For the subchondral section, lower correlations were achieved for the parameters Tb.Sp and Tb.N for all scanners (e.g. for CBCT Tb.Sp, $R^{2}=0.58$ and $R^{2}=0.77$ for the subchondral and standard section, respectively) and similar correlations for BV/TV. For Tb.Th, higher correlations were obtained for CBCT (e.g. $R^{2}=0.83$ and $R^{2}=0.69$ for the subchondral and standard section, respectively), but this was not the case for XtremeCT and XtremeCT-II.

\section{Bone Mechanical Parameters}

All scanners had a high accuracy $\left(R^{2} \geq 0.96\right.$ for stiffness as well as for strength-Fig. 4). For stiffness of the standard section, the offset varied between $9.1 \%$ (XtremeCT-II) and $24.6 \%$ (CBCT). XtremeCT and XtremeCT-II performed slightly better $\left(R^{2}=0.98\right.$ for the standard section for both scanners) than the CBCT scanner $\left(R^{2}=0.96\right)$. The trends for bone strength were similar to those for bone stiffness, but the offsets were slightly higher ( $11.2 \%$ to $28.9 \%)$.

For the subchondral section, similar trends were observed as for the standard section, but the offsets were higher (between 1.4 and $57.3 \%$ for the stiffness and between 4.9 and $64.1 \%$ for the strength).

\section{Optimization of Segmentation Parameters for CBCT}

For the CBCT images, the thresholds of the adaptive segmentation had to be optimized. For the bone microstructural parameters, the optimal low global threshold for BV/TV, Tb.Sp and Tb.N varies between 22 and $26 \%$ of the highest grey value for those parameters optimized independently for the different sections (Fig. 5). For Tb.Th, the optimal threshold was higher and between 30 and $32 \%$ of the highest grey value of the image. The specific value of the high global threshold did not affect the segmentation, because the trabecular structure did not contain thick bone structures. Hence, the low global threshold was fixed to $24 \%$ of the highest grey value for BV/TV, Tb.Sp and TB.N and to $30 \%$ for Tb.Th.

For the bone mechanical parameters of the subchondral bone section, best accuracy was obtained when a low global threshold of $24 \%$ of the highest grey value was combined
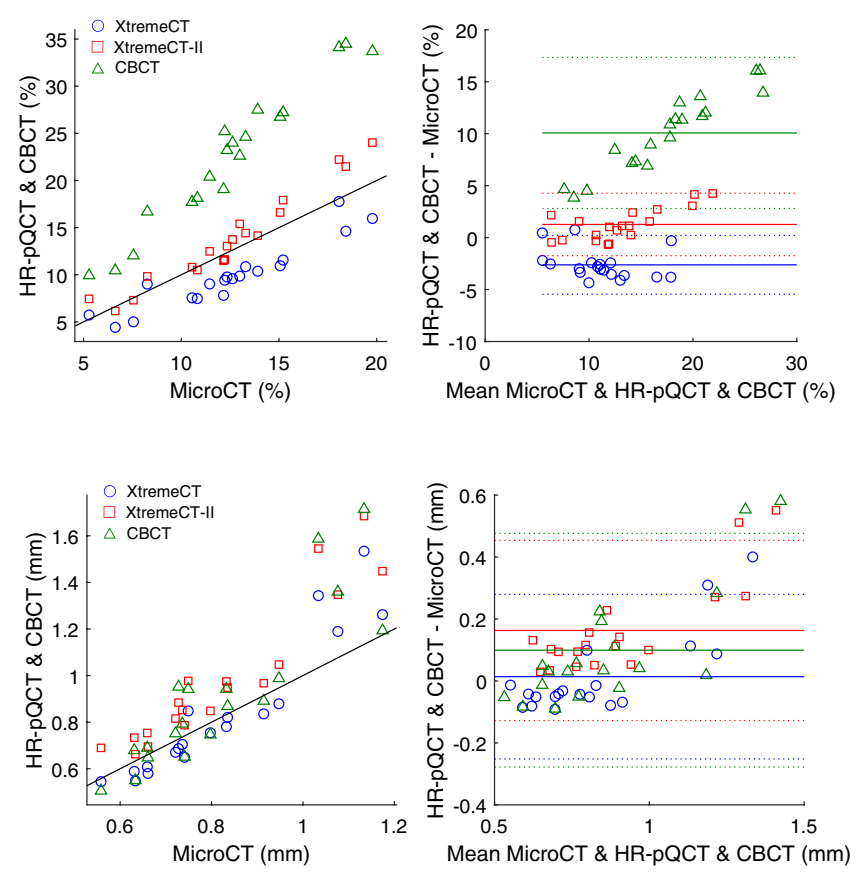

Fig. 3 Scatter plots and Bland-Altman plots between MicroCT and XtremeCT, XtremeCT-II and CBCT for the standard section for bone volume fraction (BV/TV), trabecular thickness (Tb.Th), trabecular
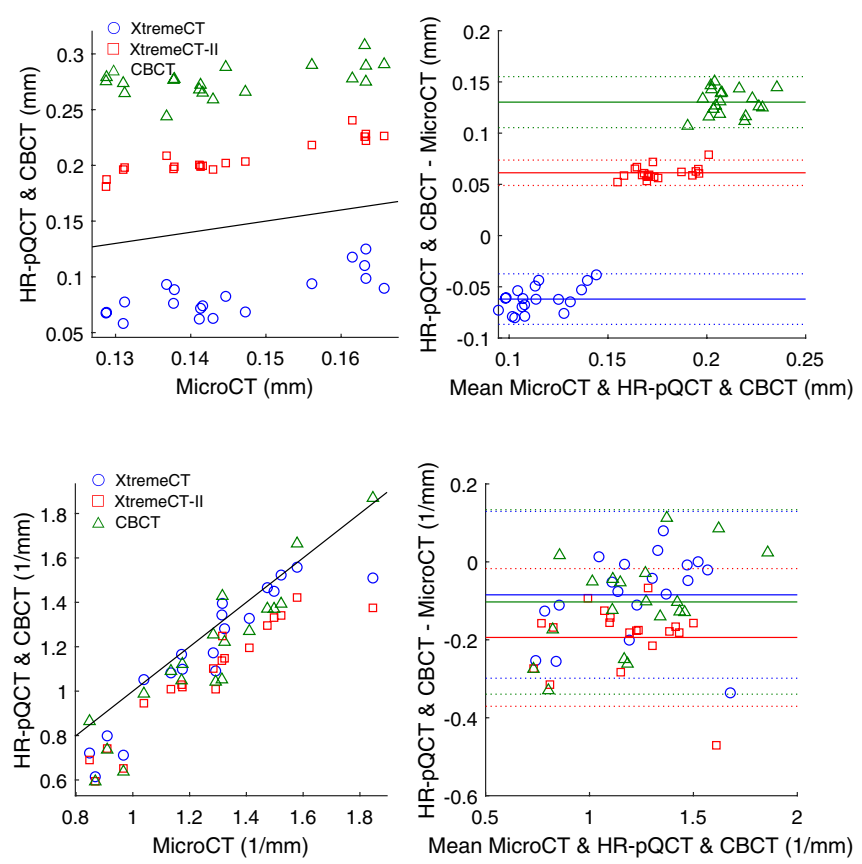

separation (Tb.Sp) and trabecular number (Tb.N). The solid line on the scatter plot indicates the line $y=x$ 
Fig. 4 Scatter plots and BlandAltman plots between MicroCT and XtremeCT, XtremeCT-II and $\mathrm{CBCT}$ for the standard section for bone stiffness and strength. The solid line on the scatter plot indicates the line $\mathrm{y}=\mathrm{x}$
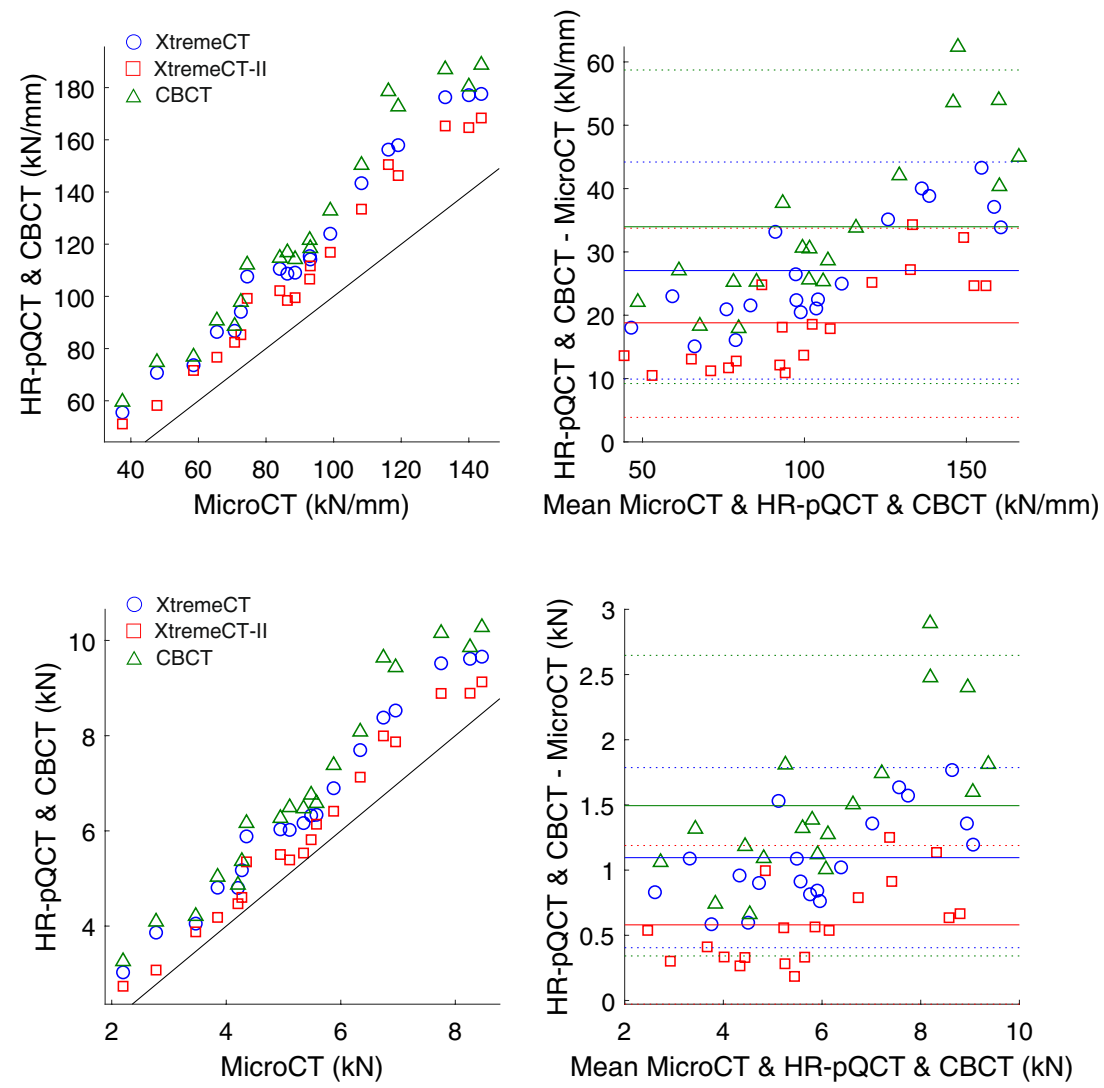

with a high global threshold of $38 \%$ of the highest grey value. For the standard bone section, the optimal values were $22 \%$ and $42 \%$, respectively. In order to standardize these settings, fixed low and high threshold values of $24 \%$ and $38 \%$ were used for the reported results of both sections.

\section{Discussion}

The XtremeCT-II images had higher physical resolution and the reconstructions appeared visually sharper and with more contrast than the CBCT images. Yet, the accuracies in bone microstructural and bone mechanical parameters obtained in this study with XtremeCT-II and with CBCT were very similar, except for Tb.Sp, which showed better accuracy with XtremeCT-II. We hypothesize that this can be explained as follows: the adaptive segmentation was in general able to capture the bone microstructure of the CBCT images with a high accuracy, but it was not able to detect all the small trabeculae which mainly influence the parameter Tb.Sp. The inability to detect small trabeculae is more pronounced on the CBCT scans, but also HR-pQCT has problems with it. Mainly the bones with high Tb.Sp have many of those small trabeculae. The offset of the CBCT images was higher than the offset of XtremeCT-II. It is known that a lower spatial resolution will lead to higher offsets [17], which has as disadvantage that it becomes more important to calculate correction factors.

The accuracy of XtremeCT to quantify bone microstructural parameters was, in general, slightly lower compared to the other two scanners, despite that the XtremeCT images appeared visually slightly sharper than CBCT images. However, the images of the different scanners were segmented with different approaches and this may have affected the results. In particular, to achieve high accuracy with CBCT, the results of this scanner were pushed to the limits by means of software and CBCT is clearly inferior to HR-pQCT while making use of the standard reconstruction software of the CBCT scanner. We hypothesize that, by using more sophisticated segmentation approaches, the quantification accuracy could be improved for XtremeCT and potentially also for XtremeCT-II, compared to the standard method. With such optimization, the HR-pQCT scanners may achieve superior results versus CBTC. However, an actual optimization of the segmentation technique of the XtremeCT and XtremeCT-II images was out of the scope of this study. For those devices, the manufacturer's default image processing methods were used with the standard settings as these represent the tools available to the users.

To obtain good accuracy with CBCT, the reconstruction of the projection data [9] as well as the segmentation technique are critical. Yet, no standard segmentation technique 

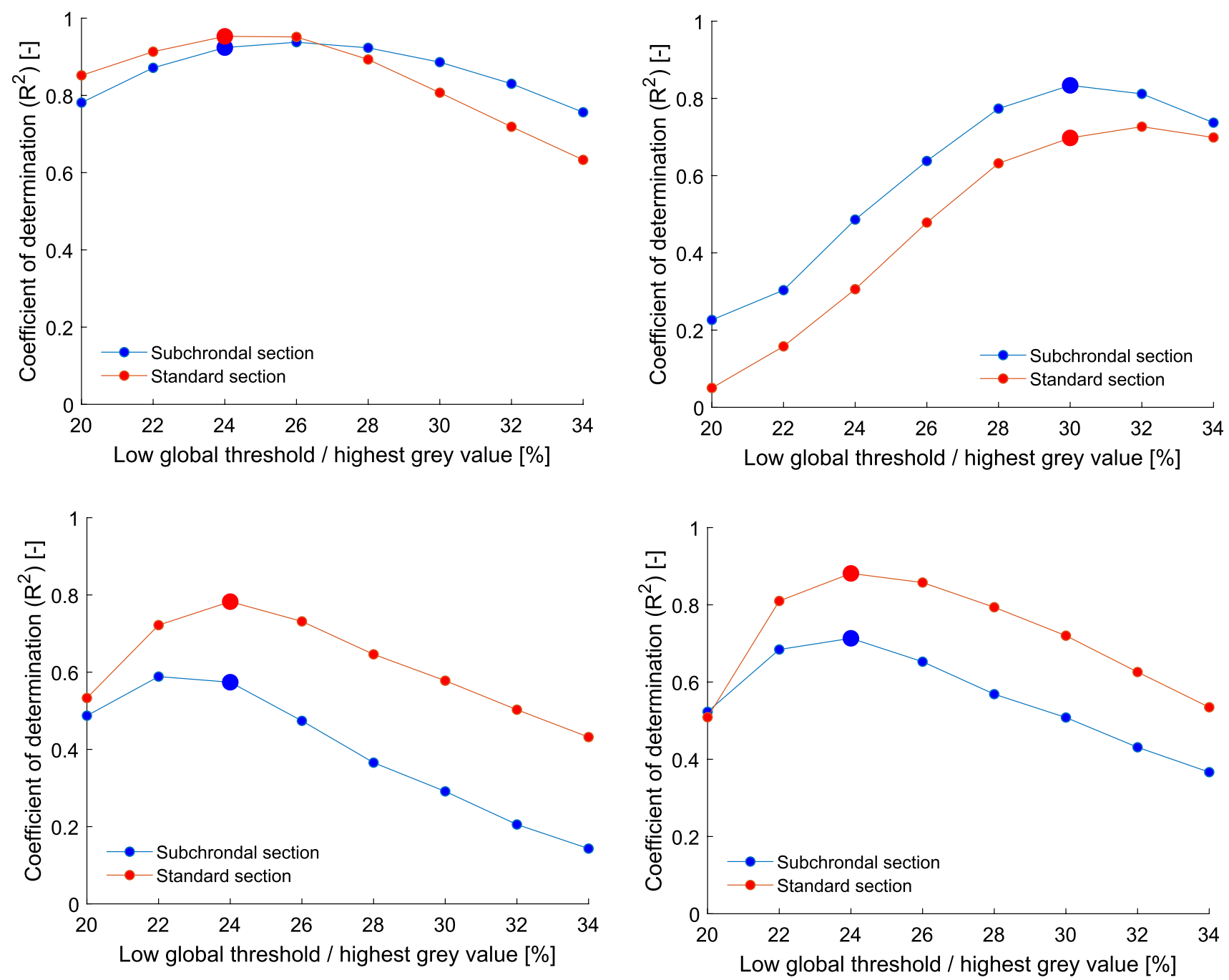

Fig. 5 Coefficient of determination $\left(R^{2}\right)$ as a function of threshold in the adaptive segmentation to quantify the bone microstructural parameters with CBCT of the subchrondal section as well as the standard section. Threshold is expressed as a percentage of the maximum grey level. For the bone microstructural parameters BV/ $\mathrm{TV}, \mathrm{Tb} . \mathrm{Sp}$ and Tb.N a low global threshold between 22 and $26 \%$ of

the highest grey value provides optimal correlation. For the Tb.Th a higher threshold between 30 and $32 \%$ of the highest grey value provides optimal correlation. The selected threshold ( $24 \%$ for BV/TV, Tb.Sp and Tb.N and 30\% for Tb.Th) is indicated on every graph with a thicker marker. Note that this does not correspond for every parameter with the highest correlation

exists for CBCT, so development of a segmentation technique as well as optimization of the segmentation parameters was needed. We used an adaptive thresholding approach in which the low global threshold was optimized to obtain highest possible correlations (Fig. 5). We found that the optimal low global threshold was significantly higher for $\mathrm{Tb}$.Th than for the other bone microstructural parameters. Therefore, we propose a dual adaptive segmentation technique for the microstructural parameters with one threshold when evaluating Tb.Th and another for BV/TV, Tb.Sp and Tb.N. This is a reasonable approach because for BV/TV, $\mathrm{Tb} . \mathrm{Sp}$ and $\mathrm{Tb} . \mathrm{N}$ it is important to quantify all trabeculae, whereas for $\mathrm{Tb}$.Th a more realistic thickness is important. For the microFE simulation, a low global threshold for the

trabecular bone structure ( $24 \%$ of the highest grey value), combined with a high global threshold for the cortex (38\% of the highest grey value) was optimal. Yet, the segmentation parameters of the microFE analyses are not critical and good agreement was reached for a broad range of thresholds (data not shown). To avoid over-optimization of the parameters, the stability of the optimization was tested over multiple random subsamples. This test showed that the chosen parameters were reasonable and stable over those subsamples (data not shown). Yet, more analyses on larger sample sizes are required to fine-tune the segmentation technique.

De Charry et al. have already demonstrated that bone microstructural parameters of distal radii determined using the NewTom 5G (Cefla, Italy) correlated well with 
XtremeCT; however, they have not evaluated the accuracy [18]. Their results cannot be directly compared with our findings as we evaluated the accuracy of the HR-pQCT and CBCT scanners against microCT. Still, in line with their findings, we also observed important offsets for most parameters between the CBCT scanner versus the XtremeCT and microCT scanner. However, it is already known that different resolutions and segmentation techniques result in different absolute values [17] and these consistent differences can be compensated for if the correction terms are known. Klintström et al. evaluated the accuracy of CBCT scanners, among other for the NewTom 5G (Cefla, Italy), against microCT to quantify bone microstructural [19] and mechanical parameters [20] on radius cubes and compared it to the accuracy obtained with XtremeCT, but not with the newest generation XtremeCT-II scanner. The correlations obtained in our study were higher than reported by Klinström et al. for all parameters except for Tb.Th. However, a direct comparison is difficult to make. In this study, we tried to mimic the measurement area recommended for clinical scanning, while the study of Klinström et al. makes use of non-further specified cubes of trabecular bone of the distal radius with a side of $8 \mathrm{~mm}$. In this study, we opted to make use of fresh-frozen bone samples. Klinström et al. made use of defatted bone samples and scanned them in water with a paraffin layer around the bone to mimic the soft tissue. We believe the fresh frozen situation is the more realistic one. According to our simulations (not shown) and reasoning, the paraffin mimics the positive effect of the soft tissue, namely reducing the beam hardening, without adding the degenerative in vivo aspects on the scan quality. In reality, the ulna will create extra artefacts and the radius is not in the centre of the scanned volume. Hence, this is the first study that evaluated the accuracy of CBCT in a clinically relevant section in the distal radius and compared it with the accuracy of XtremeCT and XtremeCT-II.

A limitation of this study was the ex vivo nature of the analyses. This means that imaging artefacts due to movement as well as due to the ulna and the surrounding soft tissue was not taken into account. Soft tissues would have an impact mainly on the beam hardening and scattering artefacts. However, the absence of the soft tissue in this study will have a negative effect on the beam hardening in the present analyses as in in vivo situations, the soft tissue acts as a filter that limits beam hardening. Motion artefacts are expected to be smaller with the shorter scanning time of CBCT versus HR-pQCT, but it has to be evaluated in future studies how these affect the images acquired with inferior resolution of CBTC compared to HR-pQCT. A second limitation is that the applied boundary conditions in the microFE-simulations do not represent realistic in vivo loading conditions. However, these are the standard boundary conditions used in other studies for microFE simulations and correspond to those applied in the standard microFE analyses of the HR-pQCT software. A third limitation is that the CBCT scanner is not calibrated to bone mineral density (BMD). And a last limitation that is assumed in this study is that the offsets are constant and hence, $R^{2}$ can be used as accuracy measurement. Larger datasets are needed to confirm this assumption.

\section{Conclusion}

We conclude that, for distal radius sections, CBCT-based microstructural and mechanical parameters calculated on our in-house-processed images have comparable accuracy to HR-pQCT-based parameters assessed with the standard methods. XtremeCT-II provides slightly higher accuracy than XtremeCT and CBCT. The accuracy of CBCT is higher for BV/TV and Tb.Th, but lower for Tb.Sp and Tb.N compared to XtremeCT.

For non-clinical research, HR-pQCT seems to be the best option, because it provides the sharpest scan, while the reduced scanning time and larger FOV make CBCT an interesting technique enabling high-resolution in vivo scanning in clinical practice. In future, new imaging modalities combining the positive aspects of HR-pQCT, CBCT as well as the image processing techniques developed for CBCT in this research, may advance this field.

Acknowledgements The authors are not compensated and there are no other institutional subsidies, corporate affiliations, or funding sources supporting this study unless clearly documented and disclosed. This research was supported by a FWO travel grant (Grant No. V438418N), KU Leuven Internal Funding (Grant No. C24/16/027) and the Swiss National Supercomputing Centre under Project ID 891. The authors would like to thank Ursula Eberli (AO Research Institute Davos, Davos, Switzerland) for assistance with the IPL software and Dieter Wahl (AO Research Institute Davos, Davos, Switzerland) for assistance with the development of scanning techniques and the development of scannerspecific holders.

Author Contributions Study design: KM, PV, FS, BG and HVL. Data collection: KM, VN, OV, CW and JVB. Data analysis: KM and PV. Data interpretation: KM, PV, FS, BG and HVL. Drafting manuscript: KM. Revising manuscript content: PV, FS, BG, VN, OV, CW, JVB and HVL. Approving final version of manuscript: KM, PV, FS, BG, VN, OV, CW, JPV and HVL. KM takes responsibility for the integrity of the data analysis.

\section{Compliance with Ethical Standards}

Conflict of interest Karen Mys, Peter Varga, Filip Stockmans, Boyko Gueorguiev, Verena Neumann, Olivier Vanovermeire, Caroline E. Wyers, Joop P.W. van den Bergh and G. Harry van Lenthe declare that they have no conflict of interest.

Human and Animal Rights and Informed Consent Nineteen human cadaveric radii (11 right, 8 left) of 14 female and 5 male donors aged between 25 to 93 years (mean \pm standard deviation $(\mathrm{SD})$ : $67.9 \pm 16.2$ 
years) were used. The specimens were obtained from Science Care (Phoenix, AZ, USA) following study approval by the institutional internal review board, based on the approval of the specimens' delivery by Science Care Ethics Committee. All donors gave their informed consent inherent within the donation of the anatomical gift statement during their lifetime.

\section{References}

1. Peacock M, Turner CH, Econs MJ, Foroud T (2002) Genetics of osteoporosis. Endocr Rev 23:303-326. https://doi.org/10.1007/ s10354-005-0249-2

2. Hernlund E, Svedbom A, Ivergård M et al (2013) Osteoporosis in the European Union: medical management, epidemiology and economic burden: a report prepared in collaboration with the International Osteoporosis Foundation (IOF) and the European Federation of Pharmaceutical Industry Associations (EFPIA). Arch Osteoporos. https://doi.org/10.1007/s11657-013-0136-1

3. Blake GM, Fogelman I (2007) The role of DXA bone density scans in the diagnosis and treatment of osteoporosis. Postgrad Med J 83:509-517. https://doi.org/10.1136/pgmj.2007.057505

4. Kanis JA, Johnell O, Oden A et al (2008) FRAX ${ }^{\mathrm{TM}}$ and the assessment of fracture probability in men and women from the UK. Osteoporos Int 19:385-397. https://doi.org/10.1007/s0019 8-007-0543-5

5. Sanders KM, Nicholson GC, Watts JJ et al (2006) Half the burden of fragility fractures in the community occur in women without osteoporosis. When is fracture prevention cost-effective? Bone 38:694-700. https://doi.org/10.1016/j.bone.2005.06.004

6. Schuit SCE, Van Der Klift M, Weel AEAM et al (2004) Fracture incidence and association with bone mineral density in elderly men and women: the Rotterdam Study. Bone 34:195-202. https ://doi.org/10.1016/j.bone.2003.10.001

7. Müller R, Hildebrand T, Rüegsegger P (1994) Non-invasive bone biopsy: a new method to analyse and display the three-dimensional structure of trabecular bone. Phys Med Biol 39:145-164. https://doi.org/10.1088/0031-9155/39/1/009

8. De Cock J, Mermuys K, Goubau J et al (2012) Cone-beam computed tomography: a new low dose, high resolution imaging technique of the wrist, presentation of three cases with technique. Skeletal Radiol 41:93-96. https://doi.org/10.1007/s0025 6-011-1198-z

9. Mys K, Stockmans F, Vereecke E, van Lenthe GH (2018) Quantification of bone microstructure in the wrist using cone-beam computed tomography. Bone 114:206-214. https://doi.org/10.1016/j. bone.2018.06.006

10. Mys K, Varga P, Gueorguiev B et al (2018) A comparative analysis between cone-beam computed tomography and high-resolution peripheral computed tomography for the quantification of bone microstructure in the wrist. J Bone Miner Res 34(5):867-874

11. Elizabeth JS, Biver E, Burt L et al (2019) Cortical and trabecular bone microarchitecture predicts incident fracture independently of DXA bone minderal density and FRAX in older women and men: the Bone Microarchitecture International Consortium (BoMIC). Lancet 7:34-43. https://doi.org/10.1016/S2213-8587(18)30308 -5 .Cortical
12. Mys K, Varga P, Gueorguiev B et al (2019) Correlation between cone-beam computed tomography and high-resolution peripheral computed tomography for assessment of wrist bone microstructure. J Bone Miner Res 34:867-874. https://doi.org/10.1002/ jbmr.3673

13. MacNeil JA, Boyd SK (2007) Accuracy of high-resolution peripheral quantitative computed tomography for measurement of bone quality. Med Eng Phys 29:1096-1105. https://doi.org/10.1016/j. medengphy.2006.11.002

14. Buie HR, Campbell GM, Klinck RJ et al (2007) Automatic segmentation of cortical and trabecular compartments based on a dual threshold technique for in vivo micro-CT bone analysis. Bone 41:505-515. https://doi.org/10.1016/j.bone.2007.07.007

15. Flaig C (2012) A highly scalable memory efficient multigrid solver for micro-finite element analyses. ETH Zurich

16. Pistoia W, van Rietbergen B, Lochmüller E-M et al (2002) Estimation of distal radius failure load with micro-finite element analysis models based on three-dimensional peripheral quantitative computed tomography images. Bone 30:842-848

17. Kothari M, Keaveny TM, Lin JC et al (1998) Impact of spatial resolution on the prediction of trabecular architecture parameters. Bone 22:437-443. https://doi.org/10.1016/S8756-3282(98)00031 $-3$

18. de Charry C, Boutroy S, Ellouz R et al (2016) Clinical cone beam computed tomography compared to high-resolution peripheral computed tomography in the assessment of distal radius bone. Osteoporos Int 27:3073-3082. https://doi.org/10.1007/s0019 8-016-3609-4

19. Klintström E, Klintström B, Moreno R et al (2016) Predicting trabecular bone stiffness from clinical cone-beam CT and HRpQCT data; an in vitro study using finite element analysis. PLoS ONE 11:1-19. https://doi.org/10.1371/journal.pone.0161101

20. Klintström E, Klintström B, Pahr D et al (2018) Direct estimation of trabecular bone stiffness using cone-beam computed tomography. Oral Surg Oral Med Oral Pathol Oral Radiol 126:72-82. https ://doi.org/10.1016/j.oooo.2018.03.014

21. Verhulp E, Van RB, Müller R, Huiskes R (2008) Indirect determination of trabecular bone effective tissue failure properties using micro-finite element simulations. J Biomech 41:1479-1485. https ://doi.org/10.1016/j.jbiomech.2008.02.032

22. Macneil JA, Boyd SK (2008) Bone strength at the distal radius can be estimated from high-resolution peripheral quantitative computed tomography and the finite element method. Bone 42:12031213. https://doi.org/10.1016/j.bone.2008.01.017

23. Graeff C, Marin F, Petto H et al (2013) High resolution quantitative computed tomography-based assessment of trabecular microstructure and strength estimates by finite-element analysis of the spine, but not DXA, reflects vertebral fracture status in men with glucocorticoid-induced osteoporosis. Bone 52:568-577. https:// doi.org/10.1016/j.bone.2012.10.036

Publisher's Note Springer Nature remains neutral with regard to jurisdictional claims in published maps and institutional affiliations. 